\title{
Öber die Guanylsäure aus der Pankreasdrüse.
}

Von

\section{H. Steudel.}

(Aus dem physiologischen Institut in Heidelberg.) (Der Redaktion zugegangen am 28. Oktober 1907.)

Die Guanylsäure, von Bang ${ }^{1}$ ) aus dem Nucleoproteid der Pankreasdrüse dargestellt, unterscheidet sich so wesentlich von den echten Nucleinsäuren, daß sie ein besonderes Interesse beansprucht. Daß gerade in der Pankreasdrüse eine den Nucleinsäuren nahestehende Substanz vorkommen sollte, die kein Adenin enthalten sollte, war schon auffallend: war doch das Adenin gerade in der Pankreasdrüse zuerst von $\mathrm{Koss} \mathrm{el}^{2}$ ) aufgefunden. Ferner mußte das Vorkommen von Glycerin unter den Spaltungsprodukten der Guanylsäure als ungewöhnlich erscheinen, da sämtliche anderen, bisher genauer untersuchten Nucleinsäuren kein Glycerin enthielten und die Guanylsäure durch diesen Bestandteil in nahe Beziehung zu den Lecithinen gerückt wäre. Sodann ließen spätere Angaben von Bang ${ }^{3}$ ) selbst seine früheren Resultate zweifelhaft erscheinen, denn nach seinen neueren Mitteilungen hat er, freilich nach einem anderen Verfahren, eine nucleinsäureartige Substanz aus Pankreas erhalten, die wesentlich von der früheren Guanylsäure abweicht. Endlich läßt sich nach Neumanns Angaben ${ }^{4}$ ) nach dem von ihm veröffentlichten Verfahren eine der Thymusnucleinsäure ähnliche Substanz aus Pankreas gewinnen, die nach Levenes ${ }^{5}$ ) Untersuchungen außer Guanin auch Adenin enthalten soll.

1) Diese Zeitschrift, Bd. XXVI, S. 133; Bd. XXXI, S. 411.

2) Ber. d. Deutsch. chem. Gesellsch., Bd. XVIII, S. 79 und 1928. Diese Zeitschrift, Bd. X, S. 250.

s) Beiträge zur chem. Physiologie, Bd. IV, S. 175.

4) Hoppe-Seyler-Thierfeld er, Handbuch d. physiol.-chemischen Analyse, 7. Auflage, S. 363.

5) Diese Zeitschrift, Bd. XXXII, S. 540; Bd. XXXVII, S. 402 ; Bd. XLI, S. 404 ; Bd. XLIII, S. 199. 
So waren denn Zweifel an der Richtigkeit der Bangschen Angaben gewiß berechtigt und ich habe vor etwa einem Jahre die Untersuchung der Guanylsäure aufgenommen. Daß die Resultate jetzt schon veröffentlicht werden, geschieht, weil v. Fürth ${ }^{1}$ ) auf Grund von Untersuchungen, die er gemeinschaftlich mit E. Jerusalem angestellt hat, zu Anschauungen gekommen ist, die sich nicht ganz mit meinen Beobachtungen decken. v. Fürth stellt die Existenz der Guanylsäure gänzlich in Abrede und meint, die Nucleinsäure des Pankreas entspräche durchaus den Nucleinsäuren der anderen Organe.

Die Ursache dafür, daß v. Fürth zu so wesentlich anderen Resultaten gekommen ist wie Bang, liegt darin, daß sich die Guanylsäure nur nach der von Bang zuerst angegebenen Methode isolieren läßt.

Es wurde nach den Angaben von Hammarsten ${ }^{2}$ ) zuerst ein Pankreasnucleoproteid bereitet. Frische Drüsen, direkt aus dem Schlachthaus bezogen, wurden zerkleinert und mit Wasser rasch zum Sieden erhitzt; nach dem Erkalten wurde filtriert ${ }^{3}$ ) und aus dem hellbernsteingelben Filtrat das Nucleoproteid durch Zusatz von 5-10\% Essigsäure ausgefällt. Die Fällung wurde zur Reinigung in Wasser gelöst und noch einmal mit Säure wieder ausgefällt. Der Niederschlag wurde abfiltriert und mit Alkohol und Äther getrocknet. Es resultierte ein feines, staubendes, weißes Pulver, von dem nach und nach etwa $75 \mathrm{~g}$ gesammelt wurden.

Zur Kontrolle wurden in dem Präparat einige Phosphorbestimmungen gemacht, der Phosphor wurde nach der Veraschung mit Soda und Salpeter als Magnesiumpyrophosphat zur Wägung gebracht.

$$
\begin{aligned}
& 0,5290 \text { g gaben } 0,0924 \mathrm{~g} \mathrm{Mg}_{2} \mathrm{P}_{2} \mathrm{O}_{7}=4,86 \% \mathrm{P} \\
& 0,5472 \text { » } 0,0884 \text { }=4,50 \% \text { » } \\
& 0,6204 \text { » } 0,1082 \text { > }=4,85 \% \text {, }
\end{aligned}
$$

Hammarsten hat in seinen Präparaten durchschnittlich $4,48 \% \mathrm{P}$ gefunden.

1) Beiträge zur chem. Physiologie, Bd. X, S. 174.

2) Diese Zeitschrift, Bd. XIX, S. 19.

3) Die Verarbeitung des Rückstandes siehe S. 543. 
Dieses Nucleoproteid wurde nach Bang s erster Vorschrift ${ }^{1}$ ) zur Darstellung der Guanylsäure in Portionen von je $12 \mathrm{~g}$ mit $400 \mathrm{ccm} \mathrm{2 \%}$ iger Kalilauge versetzt und eine halbe Stunde lang im siedenden Wasserbade gehalten, dann mit verdünnter $\mathrm{H}_{2} \mathrm{SO}_{4}$ neutralisiert und kochend heiß filtriert. Am nächsten Tage hatte sich, Bangs Angaben entsprechend, ein Bodensatz abgeschieden, der filtriert und aus heißem Wasser umgelöst wurde. Dann wurde der Niederschlag in 1\% iger Kalilauge gelöst, vom Ungelösten filtriert und zum Filtrat 5\% säure im geringen Überschuß zugesetzt, der Bodensatz filtriert, mit Alkohol gewaschen und mit Äther getrocknet. Die Ausbeute an freier Guanylsäure betrug 7,66 g, entsprechend einer Ausbeute von ca. 10\% (Bang).

Zur Identifizierung wurde an dem Präparat eine Phosphorund eine Stickstoffbestimmung gemacht.

$0,1776 \mathrm{~g}$ gaben $0,0480 \mathrm{~g} \mathrm{Mg}_{8} \mathrm{P}_{2} \mathrm{O}_{7}=7,52 \% \mathrm{P}$

$0,1756 \mathrm{~g}$ neutralisieren $22,3 \mathrm{ccm} \mathrm{n} / 10$-Oxalsäure $=17,88 \% \mathrm{~N}$ (Kjeldahl).

Bang fand in seinem Präparat ca. 7,65\% P und 18,10\% N

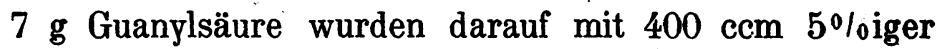
Schwefelsäure 3 Stunden lang im siedenden Wasserbade erwärmt. Nach 24 stündigem Stehen in der Kälte hatte sich eine reichliche Krystallisation langer Nadeln aus der wasserklaren Flüssigkeit abgeschieden, die durch Erwärmen wieder in Lösung gebracht wurde. Die Reaktionsflüssigkeit wurde nun einer energischen Ätherextraktion in dem von Kutscher und mir ${ }^{2}$ ) beschriebenen Apparate unterworfen und das Ätherextrakt nach Verjagen des Äthers auf Lävulinsäure geprüft. Es gelang aber weder lävulinsaures Silber zu bekommen noch fiel die empfindliche Reaktion mit Nitroprussidnatrium positiv aus. Dagegen gab das Extrakt sämtliche Reaktionen des Furfurols.

Nach der Extraktion mit Äther wurde die Zersetzungsflüssigkeit mit Natronlauge genau neutralisiert, worauf sich ein amorpher flockiger Niederschlag ausschied, der auf gewogenem Filter gesammelt wurde $(2,43 \mathrm{~g})$.

1) Diese Zeitschrift, Bd. XXVI, S. 133.

2) Diese Zeitschrift, Bd. XXXIX, S. 473.

Hoppe-Seyler's Zeitschrift f. physiol. Chemie. LIII. 
Eine Stickstoffbestimmung im Niederschlag ohne weitere Reinigung zeigt, daß es sich im wesentlichen um Guanin handelte, daß jedenfalls dem Niederschlag kein Adenin beigemengt war, denn der gefundene Wert blieb noch um 1\% hinter dem für Guanin berechneten zurück, während ein $\mathrm{Ge}-$ halt an Adenin (berechnet: 51,90\% N) sich durch eine Erhöhung der für den Stickstoff gefundenen Zahl hätte bemerkbar machen müssen.

$0,0918 \mathrm{~g}$ neutralisieren $29,8 \mathrm{ccm}$ n/10-0xalsäure $=45,45 \% \mathrm{~N}(\mathrm{Kjeldahl})$.

Berechnet für Guanin: $46,43 \% \mathrm{~N}$.

Rechnet man die Ausbeute an Rohguanin in Prozente der Guanylsäure um, so ergibt sich ein Wert von $34,72 \%$, während theoretisch 34,75\% Guanin aus der Guanylsäure hervorgehen soll. Das Filtrat vom Guanin wurde auf $250 \mathrm{ccm}$ aufgefüllt, davon wurden $10 \mathrm{ccm}$ nach Kjeldahl verascht. Sie neutralisierten bei der Destillation $4,4 \mathrm{ccm}$ n/10-Oxalsäure, enthielten also $0,00616 \mathrm{~g} \mathrm{~N}$. Im gesamten Filtrat befanden sich also noch $0,1540 \mathrm{~g} \mathrm{~N}$. Vernachlässigt man das im Filtrat sicher vorhandene Ammoniak und etwa noch unzersetzt gebliebene Guanylsäure, so würde der gesamte im Filtrat noch vorhandene Stickstoff, auf Guanin berechnet, $0,3 \mathrm{~g}$ ergeben, also eine der Hauptfällung gegenüber sehr geringe Menge. Mit pikrinsaurem Natron ließ sich keine irgendwie in Betracht kommende Fällung mehr erzielen, sodaß man wohl mit Bang $z u$ der Aussage berechtigt ist, daß in der Guanylsäure das Guanin als einziges stickstoff haltiges Spaltungsprodukt vorkommt.

Das Filtrat vom Guanin zeigte starkes Reduktionsvermögen gegen Fehlingsche Lösung, eine Identifizierung des Zuckers durch Oxydation mit Brom zu 1-Xylonsäure ') ist mir aber bisher nicht geglückt, ebenso verlief ein Versuch zum Nachweis des Glycerins gänzlich erfolglos. Daß es sich bei dem Zucker in der Guanylsäure nicht um ein Kohlehydrat der 6-Kohlenstoffreihe, auch nicht, wie v. Fürth meint, um eine Beimengung von Glykogen handelt, wird durch den Befund der Ätherextraktion bewiesen. Hier hätte unter den v. Fürth vorausgesetzten Umständen Lävulinsäure gefunden werden müssen;

') Neuberg, Ber. d. Deutsch. chem. Ges., Bd. XXXV, II., S. 1471. 
die reichliche Bildung von Furfurol und das Fehlen der Lävulin- . säure spricht mehr für eine Pentose.

So kann das Ergebnis dieses Teiles der Untersuchung dahin zusammengefaßt werden, daß aus dem Pankreasnucleoproteid von Hammarsten eine Guanylsäure gewonnen werden kann, die in ihrer Zusammensetzung im wesentlichen den Angaben Bangs entspricht. So enthält sie vor allem nur Guanin als stickstoffhaltiges Spaltungsprodukt, ferner ein Kohlenhydrat, das keine Lävulinsäure, wohl aber Furfurol liefert; dagegen ist das Glycerin kein Spaltungsprodukt der Guanylsäure.

Als die Pankreasdrüsen zum Zweck der Darstellung des Nucleoproteids mit Wasser ausgekocht worden waren, habe ich die Rückstände von der Filtration (siehe Seite 540) gesammelt und dann nach dem Neumannschen Verfahren weiter auf Nucleinsäure verarbeitet. Diese Rückstände sind dazu sehr gut geeignet, denn auch nach Neumann wird das Organ zunächst mit Wasser gekocht und dann erst weiter verarbeitet. Neumann schreibt allerdings schwach essigsaure Reaktion des Wassers vor, während die Pankreasdrüsen meist ganz schwach alkalisch reagierten, das ist aber für die Darstellung der Nucleinsäure gleichgültig.

Es ließ sich so nach Neumann eine Substanz gewinnen, die den echten Nucleinsäuren anderer Organè glich und nur ein Gelatinierungsvermögen nicht zeigte. Sie ist von mir bisher nicht genauer auf ihre Elementarzusammensetzung untersucht worden, sondern wurde sofort der Spaltung mit konzentrierter Salpetersäure ${ }^{1}$ ) unterworfen. Die nach einigen Tagen auskrystallisierten Nitrate der Alloxurbasen wurden in heißem Wasser gelöst und das Guanin durch Übersättigen mit Ammoniak ausgeschieden. Es fiel als schneeweiße Masse aus. Das Filtrat vom Guanin wurde durch Erwärmen vom überschüssigen Ammoniak befreit und mit pikrinsaurem Natron bei neutraler Reaktion ausgefällt. Nun entstand der für Adeninpikrat charakteristische hellgelbe Niederschlag, der sich schon makroskopisch von dem goldgelben Guaninpikrat unterscheidet. Das Adeninpikrat wurde noch einmal aus heißem Wasser um-

1) H. Steudel, Diese Zeitschrift, Bd. XLVIII, S. 425. 
544 H. Steudel, Über die Guanylsäure aus der Pankreasdrüse.

krystallisiert; es fielen dann zierliche Nadeln aus, die einen Schmelzpunkt von $280^{\circ}$ hatten, also den für Adeninpikrat verlangten.

Ich glaube, der Widerspruch, der in den Angaben Bangs und v. Fürths liegt, wird durch die vorstehend geschilderten Versuche gelöst. Zweifellos existiert die von Bang beschriebene Guanylsäure (freilich enthält sie kein Glycerin), daneben existiert aber noch eine echte Nucleinsäure im Pankreas, die auch Adenin in ihrem Molekül enthält. Diese letzte Substanz ist nach dem Verfahren von Neumann darstellbar, während die Guanylsäure sich nach Bangs ursprünglichen Angaben bereiten läßt. Das von Bang und Raaschou angegebene Verfahren, nach dem.v. Fürth und Jerusalem gearbeitet haben, würde dann im wesentlichen dieselbe Substanz liefern, die man nach der Neumannschen Methode gewinnen kann. 